\title{
On the Effects of Entry in Cournot Markets*
}

\author{
Rabah Amir ${ }^{\dagger}$ and Val E. Lambson ${ }^{\ddagger}$ \\ This version: September 1998
}

\begin{abstract}
In the framework of symmetric Cournot oligopoly, this paper provides two minimal sets of assumptions on the demand and cost functions that imply respectively that, as the number of firms increases, the minimal and maximal equilibria lead to (i) decreasing industry price and increasing or decreasing per-firm output; and (ii) increasing industry price and decreasing per-firm output. In both cases, per-firm profits are decreasing.

The analysis relies crucially on lattice-theoretic methods and yields general, unambiguous and easily interpretable conclusions of a global nature. As byproduct of indepensent interest, new insight into existence of Cournot equilibrium is developed.

Key Words and Phrases: Cournot oligopoly, quasi-competitiveness, supermodular games, equilibrium comparative statics.

JEL Classification Codes: C72, D43, L13

*We are grateful to William Novshek and John Roberts for helpful discussions and suggestions. We also thank Isabel Grilo, Jean-François Mertens and Xavier Vives for their perceptive comments, and two referees and the editor Hyun Song Shin for very thorough reports with many helpful suggestions. This research was initiated while both authors were enjoying the stimulating academic environment of C.O.R.E., Belgium. Financial support from C.O.R.E, and from the Ministry of Education, Science, Research and Technology, Bonn, Germany and the city-state of Berlin, is gratefully acknowledged.

†Department of Economics, Odense University, DK 5230 Odense M, Denmark; e-mail: raa@busieco.ou.dk

¥Department of Economics, Brigham Young University, Provo UT 84602, USA; e-mail: vel@email.byu.edu
\end{abstract}




\section{Introduction}

The dependence of equilibrium outputs, price and profits on industry concentration is a fundamental issue in economic analysis. Interest for this topic at various levels has traditionally extended to several subfields encompassing high theory and antitrust practice. Conventional wisdom contends that with more firms, industry price must decline. This corresponds to the so-called property of quasi-competitiveness for a Cournot oligopoly. Likewise, per-firm output and profit ought to decrease with the number of firms.

The purpose of the present paper is to thoroughly examine the validity of these assertions for a Cournot industry with symmetric firms. Our primary aim is to derive precise and minimal sufficient conditions under which these conclusions - which are often taken to be universally true - do, in fact, hold. Our findings only partly confirm conventional wisdom. In order to provide a summary, let $P(\cdot)$ and $C(\cdot)$ denote the inverse demand and the common cost function respectively. The key determinant of

the overall analysis is the sign of $\triangle \triangleq-P^{\prime}(z)+C^{\prime \prime}(x)$, where $z$ and $x$ stand for aggregate and single-firm outputs, respectively.

If $\triangle$ is globally positive, there always exists at least one symmetric equilibrium and no asymmetric ones. Furthermore, the extremal (i.e., maximal and minimal) equilibria call for a total industry output which increases in the number of firms $n$, and hence for an equilibrium price which decreases in $n$. This is the so-called property of quasi-competitiveness. As $n$ increases, per-firm equilibrium output decreases if demand is log-concave, and it increases if demand is log-convex and production costs are zero. Apart from this very last statement, the above results are perfectly intuitive and form the conventional wisdom.

On the other hand, when $\triangle$ is globally negative, two different types of Cournot equilibrium can coexist. A monopoly equilibrium always exists, where one firm produces the optimal monopoly output and the other $(n-1)$ firms produce nothing. More generally, whenever a symmetric Cournot equilibrium exists for an $m$-firm market, it remains an equilibrium for an $n$-firm market for any $n>m$, with the additional $(n-m)$ firms producing no output. These equilibria are all clearly insensitive to the 
number of firms. Given quasi-concave profit functions, a unique symmetric equilibrium also exists, which leads to per-firm and industry outputs that are decreasing in the number of firms. The latter fact, or quasi-anticompetitiveness, is highly counterintuitive, particularly given its global nature: industry price rises in response to entry by a new firm, regardless of the initial number of firms. Finally, with $\triangle<0$, we show that no other type of equilibrium exists.

A key feature of the present paper is its reliance on the lattice programming methodology. This approach allows for a clear-cut separation of the overall analysis into two mutually exclusive cases sharing no common extraneous assumptions that would have (unessential) economic meaning. This allows for a very simple and clean interpretation of the conditions (on the sign of $\Delta$ ) that drive the comparative statics conclusions: See Section 2.4. Furthermore, this simplicity and transparency of the analysis involved is nicely complemented here by the fact that the two possible global signs of $\Delta$ also separate the issue of existence of Cournot equilibrium into two natural cases.

As far as per-firm profits are concerned, conventional wisdom fully prevails. All the Cournot equilibria discussed above give rise to equilibrium profits that are nonincreasing in the number of firms.

The results of the quasi-anticompetitive case can be usefully related to some of the theories of industry structure, in particular to the theory of natural monopoly (Sharkey (1982)). This is defined as any industry with a subadditive cost function, a purely supply-side criterion. By contrast, one can present our condition $\Delta<0$ as an alternative criterion integrating demand and supply effects (through the terms $P^{\prime}$ and $C^{\prime \prime}$ respectively). Since the monopoly outcome is a Cournot equilibrium of the oligopoly here, this criterion rests on well-defined game-theoretic foundations. (This discussion is continued in Section 2.4.)

There is an extensive literature on the topics at hand, and our results have several antecedents. Existence of a symmetric equilibrium when $\triangle$ is globally positive is an interesting (though straightforward) extension of a classic result due to MacManus (1962, 1964) and Roberts and Sonnenschein (1976) and relying on convex costs. On 
the other hand, the existence question with $\triangle$ globally negative has not been analyzed previously.

As for quasi-competitiveness and profitability, several studies have developed results that overlap with ours, including MacManus (1962, 1964), Frank (1965), Ruffin (1971), Okuguchi (1973), Novshek (1980), and Seade (1980a). Since these studies relied on methods based on the Implicit Function Theorem and signing derivatives, unnecessary simplifying assumptions were typically made, such as concavity of payoffs in own output, decreasing marginal revenue, differentiability of reaction curves, etc... Furthermore, unclear or imprecise conclusions were sometimes derived, particularly in the presence of multiple Cournot equilibria (see below).

By contrast, the approach of the present paper, based on lattice-theoretic methods as developed by Topkis $(1978,1979)$, Vives (1990), Milgrom and Roberts (1990), Milgrom and Shannon (1994) and Milgrom and Roberts (1994), leads to unambiguous, consistent and meaningful statements about these issues. First, for the comparative statics questions under consideration to even make sense, any set of sufficient conditions must include provisions for a Cournot equilibrium to exist for any number of firms. Second, to account for possible multiplicity of Cournot equilibria, one must have a systematic and meaningful way to make statements about equilibrium sets or well-defined equilibrium selections, as the number of firms varies. Third, it is desirable to obtain tight comparative statics relying only on critical or fundamentally needed conditions and not on convenient assumptions imposed only by the use of an inappropriate methodology. ${ }^{1}$

For a thorough discussion of the merits of the lattice-theoretic methodology over the traditional approach along the lines raised above, the reader is referred to Milgrom and Roberts (1994). In the present context, due to the discrete nature of the parameter of interest - the number of firms - some of the disadvantages of the previous studies are magnified. For instance, consider the problem of multiple equilibria. Assume that for $n=2$ (say), there are three (symmetric) equilibria, given by the

\footnotetext{
${ }^{1}$ Such convenient assumptions often cloud the economic intuition behind the conclusions and prevent a clear-cut separation between different and potentially mutually exclusive cases of analysis (such as the cases $\Delta>0$ and $\Delta<0$ in the context at hand).
} 
intersection of the reaction curve $r(\cdot)$ with the $45^{\circ}$ line, and that for $n=3$, there are five equilibria, given by the intersection of $r(\cdot)$ with the $22.5^{\circ}$ line (see Figure 1$) .^{2}$ We prove that, as $n$ increases, the maximal and minimal equilibria call for the output by the other $(n-1)$ firms to increase, which is clearly true here. However, no meaningful statement can be made about the shift of the middle equilibrium when $n=2$ to the three middle equilibria when $n=3$. Thus, while our approach yields clear global statements about the extremal equilibria, the traditional approach gave rise to local statements that are not always well-defined. Moreover, the latter also have the drawback of requiring exact knowledge of the equilibrium point under consideration as they are expressed via an inequality condition at that point. However, with such knowledge, the comparative statics could also be checked directly by evaluating the variation of the equilibrium point with respect to the number of firms.

In the context of Cournot oligopoly, the two extremal equilibria enjoy particular welfare properties. The largest [smallest] equilibrium output is most [least] preferred by the consumers, but least [most] preferred by the firms. Nonetheless, under particular circumstances, one might be interested in the comparative statics of a nonextremal equilibrium. To such an end, the methodology of lattice programming may or may not be invoked in a local sense (see Milgrom and Roberts (1994) for more on this).

This paper is organized as follows. Section 2 provides a description of the basic model, a statement of our results, and several illustrative examples, followed by a discussion relating our results to the theory of industry structure. Section 3 contains the proofs of this paper. Finally, a very simple and self-contained review of the lattice-theoretic notions and results needed here forms the Appendix.

\section{The Model and the Results.}

This section provides a description of the Cournot model with identical firms, and a statement of all our results. The fundamental questions under consideration here can

\footnotetext{
${ }^{2}$ We argue later on that with $n$ firms, a symmetric equilibrium corresponds to an intersection of the (common) reaction curve $r(Y)$ and the line $\frac{Y}{n-1}$ (see proof of Theorem 2.5).
} 
be simply phrased as follows: How do total equilibrium output (and hence industry price), and per-firm profit and output vary with the number of firms in the industry? Alternatively, what are the effects of entry on equilibrium outputs and profits?

We consider these fundamental questions in the framework of equilibrium comparisons (as in Milgrom-Roberts (1994)), the exogenous parameter being the number of (identical) firms in the industry. The methodology for equilibrium comparison evoked here rests on the fundamental results from supermodular optimization/games: Topkis (1978, 1979), Milgrom-Roberts (1990,1994), Sobel (1988) and Milgrom-Shannon(1994). In particular, we build on the results of Amir (1996a) which relate supermodularity analysis and Cournot oligopoly (also see Amir and Grilo (1997).)

We begin with some basic notation and definitions. A symmetric Cournot oligopoly is fully described by the quadruplet $(P, C, K, n)$ where $P: R^{+} \rightarrow R^{+}$is the inverse demand function, $K>0$ is the production capacity of each firm, $C:[0, K] \rightarrow R^{+}$is the cost function (common to all firms), and $n$ is the number of firms in the industry. (Here, $R^{+}$stands for the nonnegative reals.)

Let $x$ denote the output variable for the firm under consideration, and let $y$ be the total output variable for the remaining $(n-1)$ firms. The variable $z$ will stand for cumulative industry output, i.e., $z=x+y$. In view of the (possible) nonuniqueness of Cournot equilibria in our setting, the following notation is needed. Let $X_{n}, Y_{n}, Z_{n}, P_{n}$ and $\Pi_{n}$ denote the following sets respectively, given that the industry is comprised of $n$ identical firms: equilibrium outputs for a single firm (at a symmetric equilibrium), equilibrium total output for the remaining $(n-1)$ firms, equilibrium total output, equilibrium price, and equilibrium (per-firm) profit. Whenever any of these sets is a singleton, it will be denoted by the corresponding lower-case letter.

The profit function of the firm under consideration is

$$
\Pi(x, y)=x P(x+y)-C(x)
$$

Alternatively, one may think of the firm as choosing total output $z=x+y$, given the other firms' cumulative output $y$, in which case its profit is given by

$$
\widetilde{\Pi}(z, y)=\Pi(z-y, y)=(z-y) P(z)-C(z-y)
$$


Let $\triangle(z, y)$ denote the cross-partial derivative of $\widetilde{\Pi}$ with respect to $z$ and $y$,

$$
\triangle(z, y)=-P^{\prime}(z)+C^{\prime \prime}(z-y)
$$

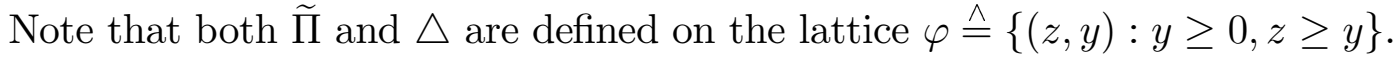

The following Standard Assumptions are in effect throughout the paper:

(A1) $P(\cdot)$ is continuously differentiable and $P^{\prime}(\cdot)<0$.

(A2) $C(\cdot)$ is twice continuously differentiable and nondecreasing.

Note that, although convenient, the smoothness assumptions are by no means necessary for our main results, as will be argued below.

Whenever well-defined, denote the maximal and minimal points of a set by an upper and a lower bar, respectively. Thus, for instance, $\bar{Z}_{n}$ and $\underline{Z}_{n}$ are the highest and lowest total equilibrium outputs, with corresponding equilibrium prices $\underline{P}_{n}$ and $\bar{P}_{n}$, respectively. Performing comparative statics on equilibrium sets will consist of predicting the direction of change of these extremal elements as the exogenous parameter varies. Accordingly, the usual notion of quasi-competitiveness from models with a unique equilibrium (Ruffin (1971)) is extended as follows:

Definition. A symmetric Cournot model is said to be quasi-competitive [quasianticompetitive] if the extremal equilibrium total outputs $\bar{Z}_{n}$ and $\underline{Z}_{n}$ are nondecreasing [nonincreasing] in $n$.

The two mutually exclusive assumptions on $P$ and $C$ that respectively establish quasi-competitiveness and quasi-anticompetitiveness are also those needed to separate the issue of existence of a Cournot equilibrium into two distinct cases: $\Delta>0$ globally on $\varphi$, and $\Delta<0$ globally on $\varphi$. (The comparative statics of per-firm output $x_{n}$ requires additional information.) Consequently, it is convenient to consider two distinct cases. 


\subsection{The Quasi-Competitive Case.}

Here, we provide conditions on $P(\cdot)$ and $C(\cdot)$ ensuring that (i) a symmetric Cournot equilibrium exists for each $n=1,2, \ldots$, and (ii) the model is quasi-competitive, and leads to extremal equilibrium profits which are nonincreasing in $n$. These properties are widely believed to hold very generally - and indeed the conditions we provide are very natural - although, as will be seen in Section 2.2, there are also plausible conditions under which quasi-competitiveness fails to hold.

We begin with the existence result.

Theorem 2.1 In addition to the Standard Assumptions, suppose that $\triangle(z, y)>0$ on $\varphi$. Then, for each $n \in N$, the Cournot oligopoly has at least one symmetric equilibrium and no asymmetric equilibria.

This theorem extends the classic McManus $(1962,1964)$ result which assumes the convexity of $C$ instead of $\triangle>0$, and which was also independently rediscovered by Roberts and Sonnenschein (1976); see also Amir (1996a) for an alternative proof, based on lattice-theoretic arguments. Since $P^{\prime}<0$, it is clear that the convexity of $C$ implies $\triangle>0$ on $\varphi$. An example is provided below illustrating that the conditions of Theorem 2.1 can hold even when the cost function is everywhere concave, thereby establishing that the extension embodied in our result here is meaningful.

It is important to note that differentiability of the demand and cost functions is assumed purely for convenience (and for ease of interpretation of the conditions on $\Delta)$. As will become apparent in the proofs, the fundamentally needed assumption is the supermodularity of $\widetilde{\Pi}$ on $\varphi$ (which, under smoothness conditions is equivalent to $\Delta \geq 0,{ }^{3}$ by Topkis's Characterization Theorem: see Appendix). Equivalently, all we

\footnotetext{
${ }^{3}$ Furthermore, we would need to assume that $\widetilde{\Pi}$ is strictly supermodular on $\varphi$ to obtain the monotonicity of every best-reply selection (as opposed to the two extremal selections only: see Topkis's Theorem in Appendix), which is weaker than $\Delta>0$ on $\varphi$ (under smoothness assumptions).
} 
definitely need is ${ }^{4}$ (dropping the term $z P(z)$ of $\left.\widetilde{\Pi}\right)$ :

$$
y P(z)+C(z-y) \text { is submodular on } \varphi \text {. }
$$

The key consequence of this assumption is that the line segment joining any two points on the graph of the reaction correspondence of a firm must have a slope $\geq-1$, which in particular precludes downward jumps (while allowing for upward jumps).

We are now ready for the main result of this section.

Theorem 2.2 Under the hypothesis of Theorem 2.1, the following hold:

(a) The extremal equilibrium cumulative outputs of $(n-1)$ firms, $\bar{Y}_{n}$ and $\underline{Y}_{n}$, are nondecreasing in $n$.

(b) The extremal equilibrium total outputs $\bar{Z}_{n}$ and $\underline{Z}_{n}$ are nondecreasing in $n$, and hence the corresponding prices $\underline{P}_{n}$ and $\bar{P}_{n}$ are nonincreasing in $n$.

(c) The extremal equilibrium profits $\bar{\Pi}_{n}$ and $\underline{\Pi}_{n}$ are nonincreasing in $n$.

Rephrasing these conclusions in terms of per-firm equilibrium outputs $X_{n}$, Parts (a) and (b) would say that $n \bar{X}_{n}, n \underline{X}_{n},(n-1) \bar{X}_{n}$ and $(n-1) \underline{X}_{n}$ are nondecreasing in $n$. Thus the Cournot model is quasi-competitive here. Note that $\underline{X}_{n}$ is the Pareto-dominant Cournot equilibrium for the firms while $\bar{X}_{n}$ is the Pareto-preferred equilibrium for the consumers. Theorem 2.2 contains no information about how these extremal values of $X_{n}$ vary with $n$. Both directions of change are possible, depending on whether the reaction correspondences are downward or upward sloping: Theorems 2.3 and 2.4 provide sufficient conditions for the two cases, respectively.

Theorem 2.3 In addition to the hypothesis of Theorem 2.1, assume that $P(\cdot)$ is log-concave. Then, there exists a unique and symmetric Cournot equilibrium, with per-firm equilibrium output $x_{n}$ nonincreasing in $n$.

\footnotetext{
${ }^{4}$ Alternatively, letting $D$ denote the direct demand function so that $p=P(z)$ iff $z=D(p),(2.4)$ can also be expressed as: $y p+C[D(p)-y]$ having nondecreasing differences on $\{(y, p): y \leq D(p)\}$. With smoothness, this becomes $C^{\prime \prime}[D(p)-y] D^{\prime}(p) \leq 1$.
} 
The fact that the $\log$-concavity of $P(\cdot)$ is sufficient to yield downward-sloping reaction correspondences, and that uniqueness of Cournot equilibrium (without the symmetry assumption) follows from the additional condition of convex costs, have been proved in Amir (1996a). The uniqueness part of Theorem 2.3 may thus be viewed as an extension of the latter result as it replaces the assumption of convex costs with the assumption $\Delta>0$. In view of these facts, it becomes obvious that Theorem 2.3 follows directly from Theorem 2.2(a) since $x_{n}$ is the best-response to $y_{n}$ here. Next, we state the result for the case of upward-sloping reactions.

Theorem 2.4 In addition to the hypothesis of Theorem 2.1, assume that $P(\cdot)$ is log-convex and that $C(\cdot) \equiv 0$. Then the extremal equilibrium per-firm outputs $\bar{X}_{n}$ and $\underline{X}_{n}$ are nondecreasing in $n$.

There are some major differences between the two cases. First, the presence of (nondegenerate) production costs favors downward-sloping reactions, so that the condition of zero costs is actually needed here (in particular, for the case of linear costs $c x$, Amir (1996a) shows that $P(\cdot)-c$ cannot be a log-convex function, in nondegenerate cases). Furthermore, while log-convexity is, in some sense, the dual notion to log-concavity, the latter notion is much more widely satisfied as a condition imposed on a demand function. It is easily seen via examples that log-convexity is a rather stringent requirement. These points are discussed in great detail in Amir (1996a). Finally, note that the hypothesis of Theorem 2.4 does not necessarily lead to a unique Cournot equilibrium, whence the need to specify the extremal selections again.

We conclude this subsection with three illustrative examples. The first shows that our results here can hold even when the cost function is everywhere concave. Recall that the classic antecedent of the existence part of our results here required a cost function which is everywhere convex (McManus (1964) and Roberts-Sonnenschein (1976)). 
Example 1 Consider a symmetric Cournot oligopoly with the inverse demand and cost functions given by

$$
P(z)=\left\{\begin{array}{ll}
2-z & , \quad z \leq 2 \\
0 & , \quad z \geq 2
\end{array} \text { and } C(x)=\log (x+1), x \geq 0 .\right.
$$

Assume that there are no capacity constraints here. The profit function is

$$
\Pi(x, y)=x(2-x-y)-\log (x+1), x, y \geq 0, x+y \leq 2 .
$$

The first-order condition for $\max _{x} \Pi(x, y)$, given $y \in[0,2]$, is $2-2 x-y-\frac{1}{x+1}=0$, which reduces to $2 x^{2}+x y+y-1=0$. Solving this quadratic equation, one arrives at the following best-response function and monopoly output $x^{M}$ :

$$
r(y)=\left\{\begin{array}{ll}
\frac{1}{4}\left[-y+\sqrt{y^{2}-8 y+8}\right] & , \text { if } y \leq 1 \\
0 & , \text { if } y \geq 1
\end{array} \text { and } x^{M}=r(0)=\frac{\sqrt{2}}{2} .\right.
$$

It is left to the reader to verify that $r^{\prime}(y) \geq-1$ for $y \in[0,1]$. Note also that $\Pi$ is concave in $x$ for fixed $y$ (in the relevant domain).

To compute per-firm equilibrium output, one solves the equation $x=r[(n-1) x]$, which leads to the quadratic equation $(n+1) x^{2}+(n-1) x-1=0$, whose only valid root is

$$
x_{n}=\frac{1}{2(n+1)}\left[1-n+\sqrt{n^{2}+2 n+5}\right], n \geq 1 .
$$

Then, clearly,

$y_{n}=\frac{n-1}{2(n+1)}\left[1-n+\sqrt{n^{2}+2 n+5}\right]$ and $z_{n}=\frac{n}{2(n+1)}\left[1-n+\sqrt{n^{2}+2 n+5}\right]$.

It is left to the reader to verify that $x_{n}$ is decreasing in $n$ while $y_{n}$ and $z_{n}$ are both increasing in $n$. In particular, this specification leads to a quasi-competitive outcome in spite of the concavity of $C$. The key determinant is of course that $\triangle(z, y)=-P^{\prime}(z)+C^{\prime \prime}(z-y)=2-\frac{1}{(z-y+1)^{2}}>0$ for $0 \leq y \leq z \leq 2$.

Finally, since $P$ is clearly log-concave, this example satisfies Theorem 2.3 and not Theorem 2.4.

The remaining examples are of interest from a quantitative standpoint. The next example shows that, in the context of Theorem 2.4, it is possible for $\frac{\pi_{1}}{\pi_{2}}$ and $\frac{x_{2}}{x_{1}}$ to be arbitrarily large. 
Example 2 Consider the symmetric Cournot oligopoly with no production costs, and inverse demand given by

$$
P(z)=\frac{1}{(z+1)^{\alpha}}, z \geq 0, \alpha>1
$$

The reaction curve is $r(y)=\frac{y+1}{\alpha-1}$, for $y \geq 0$. The unique (symmetric) Cournot equilibrium output, total equilibrium output and per-firm profit are respectively,

$x_{n}=\left\{\begin{array}{lll}\frac{1}{\alpha-n} & \text { if } \quad \alpha>n \\ +\infty & \text { if } \quad \alpha \leq n\end{array} z_{n}=\left\{\begin{array}{lll}\frac{n}{\alpha-n} & \text { if } \quad \alpha>n \\ +\infty & \text { if } \quad \alpha \leq n\end{array} \pi_{n}=\left\{\begin{array}{lll}\frac{(\alpha-n)^{\alpha-1}}{\alpha^{\alpha}} & \text { if } & \alpha>n \\ 0 & \text { if } & \alpha \leq n\end{array}\right.\right.\right.$

It is easily verified that the ratio of monopoly to (per-firm) duopoly profits $\frac{\pi_{1}}{\pi_{2}}$ converges to $+\infty$ as $\alpha \rightarrow 2$, and that $\frac{x_{2}}{x_{1}}$ converges to $+\infty$ as $\alpha \rightarrow 2$ (note that $x_{n}$ is increasing in $n$, as in Theorem 2.4).

The last example relates to both Theorems 2.3 and 2.4 and shows that strict monotonicity of $x_{n}$ in $n$ does not hold (without additional hypothesis).

Example 3 Consider a symmetric Cournot oligopoly with no production costs and inverse demand function

$$
P(z)=e^{-z}, z \geq 0 .
$$

Following a simple computation, we have $x_{n}=1, y_{n}=n-1$ and $z_{n}=n$, for all $n$. The reaction function of a firm is $r(y)=1, \forall y \geq 0$, so that each firm has a dominant strategy of unit output. Thus, this example fits Theorems $2.1-2.4$.

\subsection{The Quasi-Anticompetitive Case.}

In this subsection, we provide (i) conditions on $P(\cdot)$ and $C(\cdot)$ ensuring that a Cournot equilibrium, with one firm as a monopoly and the other firms not producing, always exists, and (ii) stricter conditions under which a unique symmetric Cournot equilibrium always exists. Furthermore, whenever a symmetric equilibrium for an $m$-firm oligopoly exists, it is unique (say with per-firm output $x_{m}$ ), and the following output configuration is always an equilibrium of the $n$-firm market (for all $n>m$ ): $m$ firms each produce $x_{m}$ and the other $(n-m)$ firms produce nothing. All the asymmetric 
equilibria here are (clearly) insensitive to the number of firms in the industry. On the other hand, the symmetric equilibrium reflects quasi-anticompetitiveness but still leads to per-firm profits that are decreasing in the number of firms.

Naturally, quasi-anticompetitiveness is quite counter-intuitive. Most observers of market behavior would think of equilibrium prices that increase in the number of sellers as pathological features. Nonetheless, although the associated assumptions are easily seen to be rather restrictive, it is interesting to note that the above conclusions can hold under a set of assumptions which is not degenerate. For a related result, also see Rosenthal (1980).

For the case at hand, it will be convenient (though not essential) to assume that $K=+\infty$, i.e., no capacity constraint. As will become apparent in the proofs (in Lemmas 3.1-3.2), it would be sufficient to assume $K \geq x^{M}$ ( $x^{M}=$ monopoly output).

Let $\bar{y} \triangleq P^{-1}\left(C^{\prime}(0)\right)$ and $A(\cdot)$ be the Average Cost curve, i.e., $A(x)=\frac{C(x)}{x,}, x>0$. By l'Hospital's rule, we have $P(\bar{y})=C^{\prime}(0)=A(0)$, so that $\bar{y}$ is that output by the other firms that equates price and average cost when the responding firm produces nothing. We are now ready for the existence result.

Theorem 2.5 In addition to the Standard Assumptions, suppose that $\triangle(z, y)<0$ on $\varphi$. Then, for any number of firms $n \in N$, the following hold:

(a) For any $m<n$, whenever a symmetric equilibrium exists for the $m$-firm oligopoly, it must be unique (say with output $x_{m}$ ), and the following output configuration constitutes an equilibrium for the $n$-firm oligopoly: Each of any $m$ firms produces $x_{m}$ while the remaining $(n-m)$ firms produce nothing. In particular (with $m=1$ ), an $n$-firm equilibrium always exists in which one firm produces the optimal monopoly output and the other $(n-1)$ firms produce nothing. ${ }^{5}$

\footnotetext{
${ }^{5}$ Such an equilibrium can be characterized as follows: With (say) Firm 1 as the monopoly, $x_{n}^{1}=z_{n}$ and $\sum_{i \neq 1} x_{n}^{i}=0$ for all $n$ (with superscripts indexing firms). Symmetry imposes, in the case of $n$ firms, that each possible permutation of this output vector constitute an equilibrium. Hence, there would be $n$ such monopoly equilibria.

In total, for a fixed pair $(n, m)$ with $m<n$, there are $\left(\begin{array}{l}n \\ m\end{array}\right)$ asymmetric equilibria. Hence, for
} 
(b) A unique symmetric Cournot equilibrium exists if $\Pi(x, y)$ is strictly quasiconcave in $x$ for every $y \in[0, \bar{y}]$.

(c) No other Cournot equilibrium (than those of Parts (a) and (b)) can exist.

As in the other case, we remind the reader that smoothness assumptions are only made for convenience. All we really need to have (for Parts (a) and (c)) is

$$
y P(z)+C(z-y) \text { is supermodular on } \varphi .
$$

Furthermore, in the present case, this relaxation of a convenient assumption has important economic content in that it makes it clear that (avoidable) fixed-costs in production are allowed here. ${ }^{6}$

As will be seen in the proofs, the key consequence of the assumption $\triangle(z, y)<0$ (in conjunction with the structural properties of the Cournot model) is that the interior part of the best-response correspondence (i.e., the part of the graph with $>0$ values) has all its slopes bounded above by -1 . This means that as the joint output of the other $(n-1)$ firms is increased, the firm under consideration optimally reacts by contracting its output so much that the resulting total output decreases. Hence, the best-responses are (strongly) decreasing. Thus, for $n=2$, the Cournot oligopoly is a supermodular game (upon reversal of one firm's output ordering), independently of the symmetry of the game. This is no longer true for $n \geq 3$ (see Amir (1996a)).

A sufficient condition to ensure the existence of a symmetric equilibrium for all $n$ here is the quasi-concavity of a firm's profit function in own output. This would lead to the best-response being a (single-valued) continuous function. Without this a fixed $n$, there is a total of $\sum_{m=1}^{n}\left(\begin{array}{l}n \\ m\end{array}\right)$ of these equilibria that are possible.

${ }^{6}$ Indeed, it is easy to see that if $C(0)=0$ and $C(x)>\varepsilon>0$ for $x>0$ and some $\varepsilon>0,(2.5)$ is more easily satisfied, since it requires in effect strong concavity of $C(\cdot)$. On the other hand, such a fixed-cost would typically destroy the quasi-concavity of the profit function in own output, and might thus lead to nonexistence of the symmetric equilibrium for some values of $n$. Note that in such a case, our equilibrium comparisons might be rephrased as follows: $x_{n}$ decreases in $\mathrm{n}$, for those $\mathrm{n}$ for which a symmetric Cournot equilibrium exists. 
condition, the best-response may have a downward jump where it skips over the $45^{\circ}$ line, thus implying the absence of a symmetric equilibrium in the duopoly case $(n=2)$. A similar remark can be made for $n \geq 3$, too.

The comparative statics of the equilibrium outcomes as $n$ varies is as follows.

\section{Theorem 2.6}

(a) Under the hypothesis of Theorem 2.5 (a), all the asymmetric Cournot equilibria for all $m<n$ are invariant in $n$, in the sense that all entering firms produce zero.

(b) Under the hypothesis of Theorem 2.5 (b), the symmetric Cournot equilibrium satisfies: Other firms' joint output $Y_{n}$ is nondecreasing in $n$, while per-firm output $X_{n}$, industry output $Z_{n}$ and per-firm profit $\Pi_{n}$ are nonincreasing in $n$. Hence equilibrium price is nondecreasing in $n$.

Since for each $m=1,2, \ldots, n-1$, a subset of $m$ firms produces the total output $m x_{m}$ and all other firms produce zero output, Part (a) holds in an obvious way. Part (b) contains all the nonintuitive results. Observe that there is no need for analogs to Theorems 2.3 and 2.4 here since $x_{n}$ is always strongly decreasing in $n$, which follows from the fact that $z_{n}=n x_{n}$ is nonincreasing in $n$. In view of the counter-intuitive nature of these results, an example illustrating the various points is highly desirable.

Example 4 Consider a symmetric Cournot oligopoly with the inverse demand and cost functions given by

$$
P(z)=\left\{\begin{array}{ll}
4-6 z & , \quad z \leq \frac{2}{3} \\
0 & , \quad z \geq \frac{2}{3}
\end{array} \text { and } C(x)=3 x-3 x^{2}-3 x^{3}, x \leq K=\frac{1}{3}\right.
$$

First, we compute, for $y \leq z \leq \frac{2}{3}$,

$$
\triangle(z, y)=-P^{\prime}(z)+C^{\prime \prime}(z-y)=-18(z-y) \leq 0 .
$$

The profit function is then

$$
\Pi(x, y)=x[4-6(x+y)]-3 x+3 x^{2}+3 x^{3}, x \leq \frac{1}{3}, x-y \leq \frac{2}{3}
$$

The reaction function and the monopoly output are: 


$$
r(y)=\left\{\begin{array}{ll}
\frac{1}{3}[1-\sqrt{6 y}] & \text { if } y \leq \frac{1}{6} \\
0 & \text { if } y \geq \frac{1}{6}
\end{array} \text { and } x^{M}=r(0)=\frac{1}{3}\right.
$$

It is easily checked that $r^{\prime}(y) \leq-1$ for $y \leq \frac{1}{6}$ (i.e., whenever $r(y)>0$ ).

Next, $X_{n}$ solves $x=r[(n-1) x]=\frac{1}{3}[1-\sqrt{6(n-1) x}]$. This leads to $X_{n}=\frac{1}{3}\left[n-\sqrt{n^{2}-1}\right], Y_{n}=\frac{n-1}{3}\left[n-\sqrt{n^{2}-1}\right]$ and $Z_{n}=\frac{n}{3}\left[n-\sqrt{n^{2}-1}\right]$.

It is easily verified that $X_{n}$ is a valid equilibrium (i.e., $0 \leq X_{n} \leq \frac{1}{3}$ ), $X_{n}$ and $Z_{n}$ are decreasing in $n$, and $Y_{n}$ is increasing in $n$. Hence, the example is in accordance with the general results of the quasi-anticompetitive case.

\subsection{The Hybrid Case.}

We have so far derived general conditions under which the symmetric Cournot oligopoly satisfies the natural properties of quasi-competitiveness and restrictive but nondegenerate conditions under which the opposite property holds. The latter case requires strongly increasing returns to scale in production, and is thus related to the literature on natural monopoly and oligopoly (e.g., Baumol, Panzar and Willig (1982)), as described below.

Naturally, there are also hybrid cases, where the determinant $\triangle$ changes signs on $\varphi$. Assuming the existence of Cournot equilibria, these cases would be characterized by a lack of monotonic relationship between the number of firms and the endogenous variables of interest (per-firm output, price level). The fact that a Cournot equilibrium may fail to exist in this case is established by counter-example by Novshek (1985). We do not have any general results regarding these hybrid cases, other than the following interesting unifying observation about the existence of Cournot equilibrium in general symmetric settings, which is a direct corollary of Theorems 2.1 and 2.5 .

Proposition 2.7 In addition to the Standard Assumptions, suppose that $\triangle(z, y) \neq 0$ for all $(z, y) \in \varphi$. Then the symmetric Cournot oligopoly has a Nash equilibrium.

We close with an illustrative example now: 
Example 5 Consider a symmetric Cournot oligopoly with

$$
P(z)=\frac{1}{z+1}, z \geq 0 \text { and } C(x)=\frac{1}{2} \log (x+1), \quad x \geq 0 .
$$

Assume no capacity constraint. It is easily checked that $\triangle(z, y)$ takes on both signs on $\varphi$. The profit function per firm is

$$
\Pi(x, y)=\frac{x}{x+y+1}-\frac{1}{2} \log (x+1), \quad x \geq 0, \quad y \geq 0 .
$$

The reaction function and monopoly output are, respectively:

$$
r(y)=\left\{\begin{array}{ll}
\sqrt{1-y^{2}} & , \text { if } y \leq 1 \\
0 & , \text { if } y \geq 1
\end{array} \quad \text { and } \quad x^{M}=1\right.
$$

Thus, for $y<1, r^{\prime}(y)>(=)(<)-1$ as $y<(=)(>) \frac{\sqrt{2}}{2}$

Per-firm output $x_{n}$ solves $x=\sqrt{1-(n-1)^{2} x^{2}}$, which leads to

$$
x_{n}=\frac{1}{\sqrt{n^{2}-2 n+2}}, y_{n}=\frac{n-1}{\sqrt{n^{2}-2 n+2}} \text { and } z_{n}=\frac{n}{\sqrt{n^{2}-2 n+2}}
$$

In particular, $z_{1}=1, z_{2}=\sqrt{2} \simeq 1.41, z_{3}=\frac{3}{\sqrt{5}} \simeq 1.34, z_{4}=\frac{4}{\sqrt{10}} \simeq 1.26 \ldots$ Hence, the model is not quasi-competitive for all $n \geq 1$ (since $z_{2}>z_{3}>\ldots$ and $P_{2}<P_{3}<$ ...). However, quasi competitiveness does hold for $n \in\{1,2\}$ only, as is easily verified. In view of this, one can observe that quasi-competitiveness holds if and only if the sequence of equilibrium outputs $x_{n}$ lies in the part of $r(\cdot)$ such that $r^{\prime}(y) \geq-1$, i.e., for $n \in\{1,2\}$.

For a thorough analysis of a general class of such hybrid cases characterized by piecewise linear reaction functions, the reader should consult Novshek (1984).

\subsection{Economic Scope and Interpretation}

As asserted before, the key advantage to using the lattice-theoretic approach, from the applied economist's standpoint, is that by casting the overall analysis in a framework of fundamentally needed assumptions, it may often allow for a better intuitive understanding of the economic driving forces behind the conclusions. In the present paper, this approach naturally led to two separate cases based upon the global sign of 
$\Delta$ only, and not on other nonfundamental assumptions that would typically be common to the two cases, such as concavity of profits in own output, decreasing marginal revenue, etc....

Under this (new) perspective, the following simple and appealing interpretation can be attributed to our main result: Industry (equilibrium) price decreases [increases] with the number of competing firms whenever inverse demand or price decreases faster [slower] at any given output level than does marginal cost at all lower output levels. (It is worthwhile to note the global nature of the condition: To check it at a fixed total output $z$, it is necessary to know marginal cost at all output levels between 0 and $z)$.

Further insight into the economic nature of this key condition can be gained by breaking the overall effect (on the sign of $\Delta$ ) into its two separate components. The first is measured by the rate of change of the price function and may thus be termed the market or demand-side effect, while the second is measured by the rate of change of marginal cost and may thus be referred to as the production efficiency or supplyside effect. The market effect always contributes to lowering industry price as the number of firms increases. On the other hand, the production efficiency effect goes in the same [opposite] direction whenever the cost function is convex [concave].

Under this perspective, the main result at hand can be succinctly reinterpreted as follows: Industry price decreases with the number of firms if the market and efficiency effects go in the same direction (which happens when costs are convex); otherwise, price decreases [increases] with the number of firms whenever the market effect is stronger [weaker] than the efficiency effect. (Which of the two effects dominates is clearly determined by the sign of $\Delta$, in a global sense.)

We now turn to a discussion of the economic scope of the conclusions of this paper. As far as the quasi-competitive case is concerned, the results are so natural and intuitive that the only aspects one can stress are the level of generality of the analysis and the easy-to-verify nature of the required condition. On the other hand, the other case can be viewed in relation to some well-known developments in the theory of industry structure, in an instructive way. 
Recall that in the theory of contestable markets, natural monopoly is defined by the subadditivity of the cost function (i.e., $C(x+y) \leq C(x)+C(y)$ for all $x, y \geq 0)$ ). In other words, a natural monopoly is one for which it is never cheaper to subdivide production across several firms than to produce the whole output in one firm (see Baumol, Panzar and Willig (1982) or Sharkey (1982)). This is clearly a purely supplyside criterion, as it implicitly applies regardless of the demand structure. By contrast, the second case here may be regarded as a possible definition of natural monopoly that integrates demand and supply effects. Such a criterion would clearly be more restrictive than the one offered by contestability theory: A natural monopoly in the former sense remains one in the latter sense, but not (necessarily) vice-versa. ${ }^{7}$

Furthermore, in the present context, this criterion rests on clearly laid out gametheoretic foundations in (at least) two different ways. First, we know from Theorem 2.5 that the natural monopoly constitutes a Cournot equilibrium (although the active firm is unspecified, since it may be any one of the $n$ firms). Second, the same situation may also be regarded as the (unique) Stackelberg equilibrium of a game with sequential moves and perfect information, ${ }^{8}$ involving an ordered line-up of the $n$ firms. The designated first-mover will obviously produce the monopoly output, and all subsequent firms will choose to remain inactive. Such a sequential framework for quantity oligopoly (with U-shaped average cost curves) has been proposed by Robson (1990).

Interestingly, similar remarks apply likewise to what may be termed natural oligopoly. According to Theorem 2.5, with $n$ firms in the market, $m$ firms producing $X_{m}$ (the symmetric Cournot equilibrium for the $m$-firm oligopoly, for any $m<n$ ) with the remaining $(n-m)$ firms inactive constitutes a Cournot equilibrium for the $n$-firm industry. Furthermore, this outcome may also be viewed as a Stackelberg equi-

\footnotetext{
${ }^{7}$ Even in the limit case (of the present framework) where inverse demand is flat, the criterion $\Delta \leq 0$ is equivalent to the concavity of the cost function $C$, which implies (but is not implied by) the subadditivity of $C$.

${ }^{8}$ We continue to use the term "Stackelberg equilibrium" from the classical oligopoly literature, although the term subgame-perfect equilibrium (of the game with sequential moves) would be more precise.
} 
librium where the $m$ firms are (simultaneous) first-movers and the remaining $(n-m)$ firms are followers or second-movers (who may move in any order after observing the first movers' actions). Clearly, the preceeding discussion does not extend to the quasi-competitive case. Indeed, with a total output of $m X_{m}$ already in the market, the $(m+1)^{\underline{s t}}$ firm would find it profitable to produce some output.

As a final part of this discussion, we now relate our conclusions to some recent developments in game theory itself. The main issue here is equilibrium selection in the quasi-anticompetitive case. It is well-known that the symmetric equilibrium is unstable in the sense of not being the limit of Cournot (best-reply) dynamics, see Seade (1980b). On the other hand, the monopoly equilibrium is locally stable (in the same sense). Plausibility of the symmetric equilibrium emerges then as a natural question, the importance of which is enhanced by the fact that it is the only equilibrium outcome leading to economically counter-intuitive results.

Interestingly, none of the standard Nash equilibrium refinements for one-shot games (such as normal form perfection, stability in the sense of Kohlberg-Mertens $(1986), \ldots$ ) could be invoked to rule out unstable equilibria (in the sense of Cournot dynamics). On the other hand, these equilibria could clearly be ruled out by some convergence criteria based on adaptive learning (Milgrom and Roberts (1991)), which includes Cournot dynamics, fictitious play, ..., or on some selection criteria developed in the theory of evolutionary games (see e.g., Kandori, Mailath and Rob (1993)).

Finally, recent behavioral studies have also addressed the issue of equilibrium plausibility/selection in the very setting of a Cournot output game. Experimental evidence suggests that the interior equilibrium (with strictly positive outputs) predicts play well if the equilibrium is stable (Holt (1995)) and poorly if the equilibrium is unstable (Cox and Walker (1997)). More precisely, the latter study is based on a Cournot duopoly with linear reaction curves with slopes $\leq-1$ (whenever interior) and reports no regular pattern of (laboratory) behavior that supports any of the three equilibria, one of which is stable and interior and the other two unstable and on the boundaries. 


\section{Proofs}

This section contains all the proofs of this paper (with a summary of the latticetheoretic facts in the Appendix). We begin by setting the relevant notation. A firm's best-response correspondence is defined as usual by (for $0 \leq y \leq(n-1) K$ )

$$
r(y)=\arg \max \{x P(x+y)-C(x): 0 \leq x \leq K\}
$$

It will often be convenient to think of a firm as choosing cumulative output $z$, given the other $(n-1)$ firms' total output $y$, instead of simply choosing its own output $x$. With $z \triangleq x+y$, the objective (3.1) can be rewritten as

$$
\max \{(z-y) P(z)-C(z-y): y \leq z \leq y+K\}
$$

The following mapping, which can be thought of as a normalized cumulative bestresponse correspondence, is the key element in dealing with symmetric equilibria for any $n$ :

$$
\begin{aligned}
B_{n}:[0,(n-1) K] & \rightarrow 2^{[0,(n-1) K]} \\
y & \rightarrow \frac{n-1}{n}\left(x^{\prime}+y\right)
\end{aligned}
$$

Here, $x^{\prime}$ denotes a best-response output level by a firm to a joint output $y$ by the other $(n-1)$ firms. It is readily verified that the (set-valued) range of $B_{n}$ is as given, i.e., if $x^{\prime} \in[0, K]$ and $y \in[0,(n-1) K]$, then $\frac{n-1}{n}\left(x^{\prime}+y\right) \in[0,(n-1) K]$. Also, a fixed-point of $B_{n}$ is easily seen to yield a symmetric Cournot equilibrium, for it must

satisfy $\bar{y}=\frac{n-1}{n}\left(\overline{x^{\prime}}+\bar{y}\right)$, or $\overline{x^{\prime}}=\frac{\bar{y}}{n-1}$, which says that the responding firm produces as much as each of the other $(n-1)$ firms.

\section{Proof of Theorem 2.1}

The cross-partial derivative of the maximand in (3.2) with respect to $z$ and $y$ is easily seen to be given by $\triangle(z, y)$, which is assumed $>0$ here. Hence, the maximand in (3.2) has strictly increasing differences on the lattice

$$
\varphi=\{(z, y): 0 \leq y \leq(n-1) K, y \leq z \leq y+K\}
$$

Furthermore, the feasible correspondence $y \rightarrow[y, y+K]$ is ascending in $y$. Hence, by Theorem A.1 (see Appendix), every selection from the $\arg \max , Z^{*}$, of (3.2) is 
nondecreasing in $y$. Since $Z^{*}(y)=x^{\prime}+y$, this is equivalent to saying that, for each fixed $n$, every selection of $B_{n}$ (as defined in (3.3)) is nondecreasing in $y$. Hence, by Tarski's fixed-point theorem (Theorem A.3), $B_{n}$ has a fixed-point, which is a symmetric Cournot equilibrium, as argued above.

Next, we show that no asymmetric equilibrium exists. To this end, it suffices to show that the mapping $y \rightarrow Z^{*}$ (the $\arg \max$ in (3.2)) is strictly increasing (in the sense that all its selections are strictly increasing). For then, to each $z^{\prime} \in$ $Z^{*}$ corresponds (at most) one $y$, such that $z^{\prime}=x^{\prime}+y$ with $x^{\prime}$ being a best-response to $y$ (see Novshek (1984)). In other words, for each total equilibrium output $z^{\prime}$, each firm must be producing the same $x^{\prime}=z^{\prime}-y$, with $y=(n-1) x^{\prime}$.

Let $\tilde{z}$ be an arbitrary (single-valued) selection of $Z^{*}$. Proceed by contradiction and assume that $\widetilde{z}\left(y_{1}\right)=\widetilde{z}\left(y_{2}\right)$ for some $y_{1}>y_{2}$ (recall that $\widetilde{z}$ is nondecreasing by the first part of this proof). Then, it is easily seen that $\widetilde{z}\left(y_{1}\right)$ and $\widetilde{z}\left(y_{2}\right)$ can w.l.o.g. be taken to be interior in the maximization of (3.2) and satisfy the first-order conditions

$$
P\left[\widetilde{z}\left(y_{i}\right)\right]+\left[\widetilde{z}\left(y_{i}\right)-y_{i}\right] P^{\prime}\left[\widetilde{z}\left(y_{i}\right)\right]-C^{\prime}\left[\widetilde{z}\left(y_{i}\right)-y_{i}\right]=0, i=1,2
$$

Since $\widetilde{z}\left(y_{1}\right)=\widetilde{z}\left(y_{2}\right) \hat{=} z,(3.4)$ implies

$-y_{1} P^{\prime}(z)-C^{\prime}\left(z-y_{1}\right)=-y_{2} P^{\prime}(z)-C^{\prime}\left(z-y_{2}\right)$, or

$-P^{\prime}(z)+\frac{C^{\prime}\left(z-y_{1}\right)-C^{\prime}\left(z-y_{2}\right)}{y_{2}-y_{1}}=0$. Since this equality holds for all $\mathrm{y} \in\left[y_{2}, y_{1}\right]$ (as a result of the fact that $\widetilde{z}(y)=z$ for all $\mathrm{y} \in\left[y_{2}, y_{1}\right]$ since $\widetilde{z}$ is nondecreasing), we can take limits as $y_{2} \rightarrow y_{1}$ to obtain $-P^{\prime}(z)+C^{\prime \prime}\left(z-y_{1}\right)=0$, a contradiction to $\triangle(z, y)>0$ on $\varphi$.

Hence, $\widetilde{z}$ is strictly increasing and an asymmetric equilibrium cannot exist.

\section{Proof of Theorem 2.2}

(a) The maximal and minimal selections of $B_{n}$ denoted $\bar{B}_{n}$ and $\underline{B}_{n}$ respectively, exist (by Topkis's Theorem). Furthermore, the largest equilibrium value of the output of $(n-1)$ firms, i.e., $\bar{Y}_{n}$, is the largest fixed-point of $\bar{B}_{n}$ (see (3.3)). Since $\frac{n-1}{n}$ is increasing in $n, \bar{B}_{n}(y)$ is nondecreasing in $n$, for every fixed $y$. Hence, from Theorem A.4, the largest fixed-point $\bar{Y}_{n}$ is also nondecreasing in $n$. A similar argument, using the selection $\underline{B}_{n}$, establishes that $\underline{Y}_{n}$ is also nondecreasing in $n$. 
(b) The fact that $\bar{Z}_{n}$ is nondecreasing in $n$ follows from the fact that $\bar{Y}_{n}$ is nondecreasing in $n$ and the fact that every selection of the arg max of (3.2) is nondecreasing (from the proof of Theorem 2.1). A similar argument applies to $\underline{Z}_{n}$.

(c) First, we observe that $\bar{\Pi}_{n}$ and $\underline{\Pi}_{n}$ are the equilibrium profit levels corresponding to the equilibrium outputs $\underline{X}_{n}$ and $\bar{X}_{n}$, respectively. This follows from the fact that the profit function of a firm, $\Pi(x, y)$, is (strictly) decreasing in $y$. Thus, $\bar{\Pi}_{n}$ is the optimal profit a firm gets by optimally reacting to $(n-1) \underline{X}_{n}$, and similarly for $\underline{\Pi}_{n}$. Hence, we have

$$
\begin{aligned}
\bar{\Pi}_{n} & =\underline{X}_{n} P\left(n \underline{X}_{n}\right)-C\left(\underline{X}_{n}\right) \\
& =\underline{X}_{n} P\left[\underline{X}_{n}+(n-1) \underline{X}_{n}\right]-C\left(\underline{X}_{n}\right) \\
& \geq \underline{X}_{n+1} P\left[\underline{X}_{n+1}+(n-1) \underline{X}_{n}\right]-C\left(\underline{X}_{n+1}\right), \text { by the Cournot property } \\
& \geq \underline{X}_{n+1} P\left[\underline{X}_{n+1}+n \underline{X}_{n+1}\right]-C\left(\underline{X}_{n+1}\right), \text { since }(n-1) \underline{X}_{n}=\underline{Y}_{n} \leq \underline{Y}_{n+1}=n \underline{X}_{n+1}
\end{aligned}
$$

from Part (a).

$$
=\underline{X}_{n+1} P\left[(n+1) \underline{X}_{n+1}\right]-C\left(\underline{X}_{n+1}\right)=\bar{\Pi}_{n+1}
$$

A similar argument for $\underline{\Pi}_{n}$ (using $\bar{X}_{n}$ ) completes the proof of Part (c)

\section{Proof of Theorem 2.3}

We first argue that uniqueness of Cournot equilibrium holds here. From [Amir (1996a), Theorem 2.1], we know that every selection of the best-response correspondence $r(\cdot)$ is nonincreasing, given that $P(\cdot)$ is log-concave (this is true for any nondecreasing left-continuous cost function). Moreover, the fact that every selection from the arg max of (3.2) is nondecreasing in $y$ (see proof of Theorem 2.1) is equivalent to the fact that every selection from $r(\cdot)$ has all its slopes bounded below by -1 , since $Z^{*}(y)=r(y)+y$. All together then, all the slopes of every selection from $r(\cdot)$ lie in $[-1,0]$. This leads to the uniqueness of Cournot equilibrium through a wellknown argument, a proof of which is given in [Amir (1996b), Lemma 2.3, p.127] in a different context (see also [Amir (1996a), Theorem 2.3]). Symmetry of this Cournot equilibrium follows from Theorem 2.1.

Now, the fact that $x_{n}$ is nonincreasing in $n$ follows from the facts that $x_{n}=r\left(y_{n}\right)$, $y_{n}$ is nondecreasing in $n$, and $r$ 's selections are all nonincreasing. This completes the 
proof of Theorem 2.3

\section{Proof of Theorem 2.4}

$>$ From [Amir (1996a), Theorem 3.2], we know that every selection from $r(\cdot)$ is nondecreasing as a consequence of the log-convexity of $P(\cdot)$ and the absence of costs (this follows from $\log \Pi(x, y)$ having nondecreasing differences in $(x, y)$ and Topkis's Theorem). Then, the fact that $\bar{X}_{n}$ and $\underline{X}_{n}$ are nondecreasing in $n$ follows from the facts that $\bar{X}_{n}=r\left(\bar{Y}_{n}\right), \underline{X}_{n}=r\left(\underline{Y}_{n}\right), \bar{Y}_{n}$ and $\underline{Y}_{n}$ are nondecreasing in $n$ (Theorem 2.2(a)), and $r$ 's selections are all nondecreasing. This completes the proof of Theorem 2.4 .

The proof of Theorem 2.5 requires two intermediate results. The first says that whenever interior, $r$ 's slopes are all below -1 (also, recall that since $K=+\infty$ here, the domain of $y$ is, a priori, $[0, \infty))$.

Lemma 3.1 Under the assumption of Theorem 2.5 (a), there holds $\frac{r_{1}-r_{2}}{y_{1}-y_{2}} \leq-1$ for all $y_{1}, y_{2} \geq 0$ and all $r_{1} \in r\left(y_{1}\right), r_{2} \in r\left(y_{2}\right)$ with $r_{1}, r_{2}>0$.

\section{Proof of Lemma 3.1}

The cross-partial of the maximand in (3.2) with respect to $z$ and $y$ is given by $\triangle(z, y)$, which is assumed $<0$ here. Hence, the maximand in (3.2) has strictly decreasing differences in $(z, y)$. However, the feasible set $[y, \infty)$ is ascending (and not descending) in $y$. Nevertheless, from Topkis's Theorem, we can still conclude that every selection of the $\arg \max , Z^{*}$, of (3.2) is nonincreasing whenever its graph is contained in a rectangle that is fully inscribed in $\varphi$, i.e., if $y_{1} \geq y_{2}, z_{1} \in Z^{*}\left(y_{1}\right)$, $z_{2} \in Z^{*}\left(y_{2}\right)$, then $z_{1} \leq z_{2}$, provided the four points $\left(y_{1}, z_{1}\right),\left(y_{2}, z_{2}\right),\left(y_{1}, z_{2}\right)$ and $\left(y_{2}, z_{1}\right)$ are all contained in $\varphi$. Call this property Rectangle Monotonicity or RM for short.

Next, we show that if $y_{0} \in Z^{*}\left(y_{0}\right)$ for some $y_{0} \geq 0$, then $Z^{*}(y)=\{y\}$ for all $y>y_{0}$ (or, in words, if the graph of $Z^{*}$ ever hits the diagonal, it coincides with it thereafter). Suppose not, i.e., there is $\widetilde{y}>y_{0}$ and $\widetilde{z} \in Z^{*}(\widetilde{y})$ such that $\widetilde{z}>\widetilde{y}$. Since 
$y_{0} \in Z^{*}\left(y_{0}\right)$, the optimal profit of responding to $y_{0}$ is $\widetilde{\Pi}\left(y_{0}, y_{0}\right)=\Pi\left(0, y_{0}\right)=0$. But $\widetilde{\Pi}\left(\widetilde{z}, y_{0}\right)=\Pi\left(\widetilde{z}-y_{0}, y_{0}\right)>\Pi(\widetilde{z}-\widetilde{y}, \widetilde{y}) \geq 0=\widetilde{\Pi}\left(y_{0}, y_{0}\right)$, since $P$ is strictly decreasing in $y$ wherever $P>0$, a contradiction to the fact that $y_{0} \in Z^{*}\left(y_{0}\right)$. Hence, we conclude that $Z^{*}(y)=\{y\}$ for all $y>y_{0}$.

Since the optimal monopoly outputs are finite (i.e., all elements of $Z^{*}(0)$ are finite), RM clearly implies that the graph of $Z^{*}$ eventually intersects the $45^{\circ}$ line, and then, as shown above, the graph of $Z^{*}$ will coincide with the $45^{\circ}$ line. Going back to the reaction correspondence $r(\cdot)$, via $r(y)=Z^{*}(y)-y$, the conclusion of Lemma 3.1 clearly follows.

Lemma 3.2 Under the assumptions of Theorem $2.5(b), r(\cdot)$ is a continuous function such that $r(y)>0$ for $y \in[0, \bar{y})$, and $r(y)=0$ for $y \geq \bar{y}$, where $\bar{y}=P^{-1}\left(C^{\prime}(0)\right)$.

\section{Proof of Lemma 3.2}

Since $\Pi$ is strictly quasi-concave in own output, $r$ must be a (single-valued) continuous function. From Lemma 3.1 (and its proof), we then know that $r$ decreases at a rate greater than one (in absolute value) from the unique monopoly output $x^{M}$ to 0 . We now show that $0 \in r(\bar{y})$. The first-order condition to $\max _{x} \Pi(x, \bar{y})$, which is also sufficient for a global maximum in view of the quasi-concavity of $\Pi$ in $\mathrm{x}$, is $P(x+\bar{y})+x P^{\prime}(x+\bar{y})-C^{\prime}(x) \leq 0$. Since $P(\bar{y}) \hat{=} C^{\prime}(0)$, it is easy to see that $x=0$ satisfies the first-order condition with equality, and hence that $\bar{y}$ is the smallest $y$ for which $r(y)=0$ (as seen in the proof of Lemma 3.1, $r(y)=0$ for all $y \geq \bar{y}$ ). This completes the proof of Lemma 3.2.

\section{Proof of Theorem 2.5}

(a) For the monopoly equilibrium or $m=1$, it suffices to show that $x^{M} \in r(0)$ and $0 \in r\left(x^{M}\right)$, for then one firm is producing its optimal monopoly output and all the other firms are best-responding with a zero output. Note that $x^{M} \in r(0)$ is true by definition of $x^{M}$. We now show $0 \in r\left(x^{M}\right)$. Suppose not. Then every point in $r\left(x^{M}\right)$ is $>0$. Let $x^{\prime}$ be such a point. We then have $\frac{x^{\prime}-x^{M}}{x^{M}-0}=\frac{x^{\prime}}{x^{M}}-1>-1$, which says that although $r\left(x_{M}\right)>0$ and $r(0)>0$, there is one slope in the graph 
of $r$ (joining images of 0 and $x^{M}$ ) which exceeds -1, a contradiction to Lemma 3.1. Hence $0 \in r\left(x^{M}\right)$. The proof for the other values of $m$ (i.e., $1<m<n$ ) is similar and left to the reader (note though that existence of a symmetric equilibrium for the $m$-firm market needs to be assumed here). This completes the proof of Part (a).

(b) An $n$-dimensional vector $(x, x, \ldots, x)$ is a symmetric Cournot equilibrium if and only if $y=(n-1) x$ satisfies $r(y)=\frac{y}{(n-1)}$, which says that each firm's best reaction is to produce as much as each of the other $(n-1)$ firms. Clearly, in view of Lemmas 3.1 and 3.2, the functions $r(\cdot)$ and $\frac{y}{(n-1)}$ have an intersection for every $n \geq 2$. Uniqueness (for each $n$ ) follows in an obvious way from the facts that the two functions are strictly decreasing (whenever interior) and increasing, respectively. This ends the proof of Part (b).

(c) Proceed by contradiction and suppose that another equilibrium (than those of Parts (a) and (b)) exists. Then it must be asymmetric and have at least 2 firms producing unequal nonzero outputs. Denote this equilibrium output vector by $\left(x^{1}, x^{2}, \ldots, x^{n}\right)$ and let $z=\sum_{i} x^{i}$ be the total output. Assume that (say) $x^{1}>x^{2}>0$ to reflect asymmetry. Define $y^{i}$ by $x^{i}=r\left(y^{i}\right), i=1,2$. We must have $x^{1}+y^{1}=$ $x^{2}+y^{2}=z$, with $y^{2}>y^{1}\left(\right.$ since $\left.x^{1}>x^{2}\right)$. But this contradicts the fact that the mapping $y \rightarrow Z^{*}=\arg \max$ in (3.2) is strictly decreasing as long as $Z^{*}>0$ (the latter point follows from an analogous argument to that of the second part of the proof of Theorem 2.1).

\section{Proof of Theorem 2.6}

(a) Since all entering firms produce no output, it is obvious that all the equilibria at hand here are invariant in $n$.

(b) We first show that $y_{n}$ is nondecreasing in $n$ (recall that the symmetric Cournot equilibrium is unique here.) Since a firm's profit function is strictly quasi-concave in its own output, $r$ and $B$ are continuous functions here. Since $\frac{n-1}{n}$ is increasing in $n, B_{n}(y)$ is nondecreasing in $n$, for each fixed $y$. Hence, the (unique) fixed-point $y_{n}$ (equilibrium joint output of $(n-1)$ firms) is nondecreasing in $n$ (by Theorem A.4).

The fact that equilibrium total output $z_{n}$ is nonincreasing in $n$ follows now from 
the fact that the mapping $y \rightarrow Z^{*}=\arg \max$ in (3.2) is nonincreasing (from the proof of Theorem 2.5(c)) and the above conclusion on $y_{n}$. The fact that per-firm equilibrium output $x_{n}$ is nonincreasing in $n$ follows from the fact that $x_{n}=r\left(y_{n}\right)$ and $r(\cdot)$ is strictly decreasing (as long as $r$ is interior).

Finally, the fact that $\pi_{n}$ is nonincreasing in $n$ follows from the proof of Theorem 2.2 (c), upon dropping the "bars" due to uniqueness here. This completes the proof of Theorem 2.6.

\section{Proof of Proposition 2.7}

If $\triangle(z, y) \neq 0$ on $\varphi$, then $\triangle(z, y)$ is either $<0$ everywhere or $>0$ everywhere, so that either Theorem 2.1 or Theorem 2.5 applies, and existence of a Cournot equilibrium follows.

\section{References}

[1] Amir, R. (1996a), "Cournot Oligopoly and the Theory of Supermodular Games", Games and Economic Behavior, 15, 132-148.

[2] Amir, R. (1996b), "Continuous Stochastic Games of Capital Accumulation with Convex Transitions", Games and Economic Behavior, 15, 111-131.

[3] Amir, R. and I. Grilo(1998), "Stackelberg vs. Cournot Equilibrium", Games and Economic Behavior, to appear.

[4] Baumol, W., J. C. Panzar and R.Willig (1982), "Contestable Markets and the Theory of Industry Structure", Harcourt Brace Jovanovich, New York.

[5] Cox, J. and M. Walker (1997), "Learning to Play Cournot Duopoly Strategies", Journal of Economic Behavior and Organization, to appear.

[6] Frank, C. R. (1965), "Entry in a Cournot Market", Review of Economic Studies", $32,245-250$. 
[7] Holt, C. (1995), "Industrial Organization: A Survey of the Results of Laboratory Experiments", in Handbook of Experimental Economics, A. Roth and J. Kagel eds., Princeton University Press.

[8] Kandori, M., G. Mailath and R. Rob (1993), "Learning, Mutation and Long-Run Equilibria in Games", Econometrica, 61, 29-56.

[9] Kohlberg, E. and J.-F. Mertens (1986), "On the Strategic Stability of Equilibria", Econometrica, 54, 1003-1037.

[10] MacManus, M. (1962), "Number and Size in Cournot Equilibrium", Yorkshire Bulletin of Economic and Social Research, 14, 14-22.

[11] MacManus, M. (1964), "Equilibrium, Number and Size in Cournot Oligopoly", Yorkshire Bulletin of Economic and Social Research, 16, 68-75.

[12] Milgrom, P. and J. Roberts (1990), "Rationalizability, Learning, and Equilibrium in Games with Strategic Complementarities", Econometrica, 58, 1255-1278.

[13] Milgrom, P. and J. Roberts (1991), "Adaptive and Sophisticated Learning in Repeated Normal Games", Games and Economic Behavior, 3, 82-100.

[14] Milgrom, P. and J. Roberts (1994), "Comparing Equilibria", American Economic Review, 84, 441-459.

[15] Milgrom, P. and C. Shannon (1994), "Monotone Comparative Statics", Econometrica, 62, 157-180.

[16] Novshek, W. (1980), "Cournot Equilibrium with free entry", Review of Economic Studies, 47, 473-486.

[17] Novshek, W. (1984), "Finding all n-Firm Cournot Equilibria", International Economic Review, 25, 61-70.

[18] Novshek, W. (1985), "On the Existence of Cournot Equilibrium", Review of Economic Studies, L II, 85-98. 
[19] Okuguchi, K. (1973), "Quasi-Competitiveness and Cournot Oligopoly", Review of Economic Studies, 40, 145-148.

[20] Roberts, J. and H. Sonnenschein (1976), "On the Existence of Cournot Equilibrium without Concave Profit Functions", Journal of Economic Theory, 13, $112-117$.

[21] Robson, A. (1990), "Stackelberg and Marshall", American Economic Review, 80, 69-82.

[22] Rosenthal, R. (1980), "A Model in which an Increase in the Number of Sellers Leads to a Higher Price", Econometrica, 48, 1575-1579.

[23] Ruffin, R. J. (1971), "Cournot Oligopoly and Competitive Behavior", Review of Economic Studies, 38, 493-502.

[24] Seade, J. (1980a), "On the Effects of Entry", Econometrica, 48, 479-489.

[25] Seade, J. (1980b), "The Stability of Cournot Revisited", Journal of Economic Theory, 23, 15-27.

[26] Sharkey (1982), The Theory of Natural Monopoly, Cambridge University Press, Cambridge, U.K.

[27] Sobel, M. (1988), "Isotone Comparative Statics for Supermodular Games", preprint, SUNY Stony-Brook.

[28] Topkis, D. (1978), "Minimizing a Submodular Function on a Lattice, Operations Research, 26, 305-321.

[29] Topkis, D. (1979), "Equilibrium Points in Nonzero-Sum n-Person Submodular Games", SIAM, Journal of Control and Optimization, 17, 773-787.

[30] Vives, X. (1990), "Nash Equilibrium with Strategic Complementarities", Journal of Mathematical Economics, 19, 305-321. 


\section{APPENDIX: A Survey of the Lattice-Theoretic Framework}

In an attempt to make this paper self-contained, we provide a summary of all lattice-theoretic notions and results invoked here, in the simplest framework for our needs. Dealing with real decision and parameter spaces results in a simple presentation. Every theorem presented here is a special case of the original.

A function $F: R_{+}^{2} \rightarrow R$ is supermodular [submodular] if, for $x_{1} \geq x_{2}, y_{1} \geq y_{2}$

$$
F\left(x_{1}, y_{1}\right)-F\left(x_{2}, y_{1}\right) \geq[\leq] F\left(x_{1}, y_{2}\right)-F\left(x_{2}, y_{2}\right) .
$$

If $F$ is twice continuously differentiable, Topkis's (1978) Characterization Theorem says that supermodularity [submodularity] is equivalent to $\frac{\partial^{2} F}{\partial x \partial y} \geq 0[\leq 0]$, for all $x, y$.

Furthermore, $\frac{\partial^{2} F}{\partial x \partial y}>0[<0]$ implies that $F$ is strictly supermodular [submodular], the latter notion being defined by a strict inequality in (1.1).

$F$ has the single-crossing property of SCP [dual SCP] in $(x, y)$ if

$$
F\left(x_{1}, y_{2}\right)-F\left(x_{2}, y_{2}\right) \geq[\leq] 0 \Rightarrow F\left(x_{1}, y_{1}\right)-F\left(x_{2}, y_{1}\right) \geq[\leq] 0
$$

It is obvious that (1.1) implies (1.2), while the converse is generally not true. Note that (1.1) is a cardinal notion, while (1.2) is ordinal. Thus, the SCP is sometimes also referred to as ordinal supermodularity. No smooth characterization of the SCP is known.

For $x \in R_{+}$, let $A(x)=\left[a_{1}(x), a_{2}(x)\right] \subset R_{+}$, with $a_{1}(\cdot)$ and $a_{2}(\cdot)$ being realvalued functions. $A(x)$ is ascending [descending] (in $x$ ) if $a_{1}$ and $a_{2}$ are nondecreasing [nonincreasing] in $x$. The following results on monotone maximizers are central to our approach.

Theorem A.1 (Topkis (1978)) If $F$ is upper semi-continuous (or u.s.c.) and supermodular [submodular], and $A(\cdot)$ is ascending [descending], then the maximum and minimum selections of $y^{*}(x) \triangleq \arg \max _{y \in A(x)} F(x, y)$ are nondecreasing [nonincreasing] in $x$. If $F$ is strongly supermodular [submodular], then every selection of $y^{*}(\cdot)$ is nondecreasing [nonincreasing].

Theorem A.2 (Milgrom-Shannon (1994)) If $F$ is u.s.c. and has the SCP $[D S C P]$, and $A(\cdot)$ is ascending, then the conclusion of Theorem A.1 holds. 
If $F$ has the strong SCP [strong DSCP], defined by (1.2) with a strict inequality on the RHS of the $\Rightarrow$ sign, then the conclusion of Theorem A.2 holds for every selection of $y^{*}(\cdot)$.

The main fixed-point theorem within this framework is due to Tarski (1955).

Theorem A.3 Let $C \subset R_{+}$be a compact interval, and $B: C \rightarrow C$ be a nondecreasing function. Then $B$ has a fixed point.

Our equilibrium comparisons rest on the following result due to Milgrom and Roberts (1990, 1994) and Sobel (1988): See Figure 2 for an illustration.

Theorem A.4 Let $C \subset R_{+}$be a compact interval, and $B_{t}: C \rightarrow C$ be a nondecreasing function $(\forall t \geq 0)$ such that $B_{t}(x)$ is also nondecreasing in $t, \forall x$. Then the minimal and maximal fixed-points of $B_{t}$ are nondecreasing in $t$.

We close with the following terminology. A function $F: R_{+} \rightarrow R$ is log-concave [convex] if the function Log $\mathrm{F}$ is concave [convex]. Similarly, a function $G: R_{+} \times R_{+} \rightarrow$ $R$ is $\log$-supermodular if $\log \mathrm{G}$ is supermodular.

A game with compact real action spaces is supermodular [ordinally supermodular] if each payoff function is supermodular [ordinally supermodular], under a specified order on each of the action spaces, and u.s.c. in own actions. Supermodularity is typically interpreted as a complementarity property: The marginal returns to increasing a player's strategy are higher if the other player uses a higher strategy. Naturally, if the order on one player's action set is reversed, supermodularity then characterizes a substitutability property in two-player games. 